\title{
El quehacer de las mujeres oficiales del cuerpo logístico en el Ejército Nacional de Colombia ${ }^{1}$
}

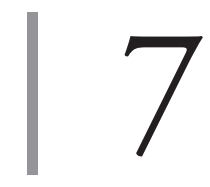

https://doi.org/10.21830/9789585318304.07

\author{
Carolina Pedraza Mariño ${ }^{2}$ \\ Diego Fernando Pérez Muñoz ${ }^{3}$ \\ Javier Ospina Anturi ${ }^{4}$ \\ Camilo Méndez Bustos ${ }^{5}$ \\ Escuela de Armas Combinadas del Ejército \\ Andrés Eduardo Fernández-Osorio ${ }^{6}$ \\ Escuela de Aviación del Ejército
}

\section{Resumen}

En este capítulo se examina el quehacer de las mujeres oficiales del cuerpo logístico en el Ejército Nacional de Colombia, a partir de la exploración de tres ejes temáticos. En primer lugar, se identificaron los orígenes de la integración de las mujeres en el Ejército y su papel en el cuerpo logístico. Posteriormente, se analizó la tradición cultural de la Fuerza respecto a las

1 Este capítulo presenta los resultados del proyecto de investigación "Construcción de los roles de la mujer militar en el posacuerdo" del Semillero de Investigación Arbitrium, Grupo de Investigación para la Capacitación Militar (GICAM), de la Escuela de Armas Combinadas del Ejército, categorizado en C por Minciencias y con código de registro COL0160714. Los puntos de vista y los resultados de este artículo pertenecen a los autores y no reflejan necesariamente los de las instituciones participantes.

2 Capitán del Ejército Nacional de Colombia. Profesional en Ciencias Militares y administradora logística (Escuela Militar de Cadetes “General José María Córdova”). Orcid: https://orcid.org/0000-0001-5055-3154 - Contacto: carolina.pedraza@buzonejercito.mil.co

3 Capitán del Ejército Nacional de Colombia. Profesional en Ciencias Militares y administrador logístico (Escuela Militar de Cadetes "General José María Córdova”). Profesional en Administración de Empresas (Universidad Nacional Abierta y a Distancia). Especialista tecnológico en Gestión del Talento Humano por Competencias (Servicio Nacional de Aprendizaje).

4 Capitán del Ejército Nacional de Colombia. Profesional en Ciencias Militares y administrador logístico (Escuela Militar de Cadetes "General José María Córdova”). Especialista en Gerencia de Proyectos (Escuela de Administración de Negocios).

5 Capitán del Ejército Nacional de Colombia. Profesional en Ciencias Militares (Escuela Militar de Cadetes “General José María Córdova”).

6 Teniente Coronel del Ejército Nacional de Colombia. PhD en Derecho y Ciencia Política (Universidad de Barcelona). Magíster en Economía, Estado y Sociedad: Política y Seguridad (University College London). Magíster en Relaciones Internacionales (Higher School of Economics Moscow). Investigador del Grupo de Investigación en Aviación Militar de la Escuela de Aviación del Ejército. Orcid: https://orcid.org/00000003-0643-0258 - Contacto: andres.fernandez@buzonejercito.mil.co 
funciones y roles de estas mujeres oficiales. Finalmente, se describió el quehacer de las mujeres oficiales del cuerpo logístico con respecto a las oficiales del cuerpo administrativo de la misma arma. Para este propósito se realizó una revisión documental sobre la incorporación de mujeres en las FF. MM. desde dos enfoques: uno tradicional y otro renovador. Los resultados permiten ampliar la comprensión del papel de las mujeres militares en el arma de Logística.

Palabras clave: Ejército Nacional; equidad de género; Fuerzas Militares; inclusión; mujer militar

\section{Introducción}

En las últimas décadas, el aumento constante de nuevas amenazas en seguridad y defensa han desafiado los gobiernos y sus Fuerzas Militares (FF. MM.), y los han obligado a mejorar sus capacidades, preparación y efectividad. La proliferación del terrorismo, las armas de destrucción masiva, el crimen organizado transnacional, la guerra cibernética y la migración ilegal, junto con una creciente competencia estratégica interestatal, han intensificado la necesidad de unas FF. MM. efectivas, que estén preparadas para enfrentar estos retos nacientes.

Este escenario, por lo tanto, ha brindado nuevas oportunidades para que el personal militar demuestre sus capacidades profesionales independientemente de su género, lo cual ha creado nuevas perspectivas para que las mujeres uniformadas desarrollen carreras estables y cumplan sus objetivos personales. En efecto, diversos académicos como Egnell et al. (2014) y Egnell y Alam (2019), así como algunas publicaciones oficiales —entre ellas, el informe de la Organización del Tratado del Atlántico Norte sobre perspectivas de género (OTAN, 2016)—, sostienen que integrar mujeres en las FF. MM. no solo cumple con las resoluciones del Consejo de Seguridad de las Naciones Unidas sobre su papel vital en la prevención y resolución de $\operatorname{conflictos}^{7}$, sino que mejora la efectividad militar y ofrece una comprensión innovadora de la seguridad y defensa.

A pesar de tales iniciativas y cambios nacionales e internacionales destinados a integrar una perspectiva de género en las FF. MM., no todos los Estados han logrado mejorar el estatus de las mujeres militares en la misma medida, y "las políticas de igualdad de oportunidades y diversidad no han tenido un éxito uniforme" (Ellner, 2015, p. 300). Aunque en 2013 el Secretario de Defensa de los Estados Unidos, Leon E. Panetta, eliminó la prohibición para las mujeres militares de servir

7 Resoluciones del Consejo de Seguridad de las Naciones Unidas N.o 1325 de octubre de 2000 y N. ${ }^{\circ}$ 1820 de junio de 2008. 
en misiones de combate (Collins-Dogrul \& Ulrich, 2018), y aunque, a partir del 2016, el 96,3 \% de los Estados miembros de la OTAN tienen todos los roles militares abiertos para las mujeres (otan, 2016), otras experiencias han mostrado múltiples dificultades para su incorporación a entornos tradicionalmente masculinos como las FF. MM. (DeGroot, 2001; King, 2016; Karazi-Presler et al., 2018; Trobaugh, 2018).

$\mathrm{Al}$ respecto, varios autores han enfatizado que los estereotipos de género en las FF. MM. son difíciles de erradicar y pueden desencadenar la disparidad en las percepciones individuales y grupales, lo que influye en opiniones y decisiones que impiden la inclusión de las mujeres en todas las especialidades militares, así como en su posibilidad para ser promovidas en su carrera (Larwood et al., 1980; Cheatham, 1984; Rohall et al., 2006; Smith \& Rosenstein, 2017).

Dado que "la prevención de conflictos, la protección de los derechos humanos y la promoción de la paz y la seguridad en todo el mundo no se pueden lograr sin la participación plena e igualitaria de las mujeres" (Verveer, 2019, p. vii), se ha publicado una cantidad considerable de estudios dedicados a este tema. Aunque extensa en cantidad, la literatura se ha centrado solo en ciertos aspectos del debate y ha dejado de lado otros temas vitales con el mismo grado de interés. Entre estos, el análisis del quehacer de las mujeres militares en ejércitos latinoamericanos y el estudio de las particularidades de las distintas armas y especialidades.

La falta de investigación sobre estos aspectos crea una brecha de conocimiento que resulta problemática tanto desde el punto de vista académico como práctico y no contribuye a la disminución de la desigualdad de oportunidades para la integración de las mujeres en el Ejército. En este contexto, el presente capítulo busca aportar a esta discusión a través de la identificación y entendimiento del quehacer de las mujeres oficiales del arma del cuerpo logístico en el Ejército Nacional de Colombia. Específicamente, se lleva a cabo una revisión documental mediante la cual se argumenta que en el contexto de inclusión en el que se ubica el Ejército Nacional, asociado a un proceso de renovación y cualificación de las Fuerzas Armadas, las mujeres militares tienen un papel importante que se debe consolidar desde las diferentes armas y especialidades en la institución.

El estudio se centró en identificar los roles y funciones de las mujeres militares del cuerpo logístico del Ejército Nacional, con el propósito de contribuir a la redefinición de su aporte a la Fuerza. Los resultados de este estudio permiten una reflexión sobre la visión instrumental de tipo tradicional que presentan las FF. MM. con respecto a la vinculación de mujeres en sus filas y sobre la necesidad de vincular una perspectiva renovadora a la luz de las dinámicas y exigencias del contexto actual para la institución militar. 


\section{Metodología}

Este estudio es de tipo descriptivo con enfoque cualitativo. Para tal fin se realizó una revisión documental orientada a explorar la historia y tendencias del personal de oficiales femeninos que integra el cuerpo logístico del Ejército Nacional. Así mismo, se buscó identificar el tipo de lineamiento que la institución busca implementar respecto a las funciones y roles de las mujeres militares. El instrumento de recolección de información fue el resumen analítico explicativo.

Se utilizaron fuentes de información de tipo secundario, como libros, archivos institucionales y otros documentos de medios digitales provenientes de las bases de datos del Ministerio de Defensa Nacional, el Ejército Nacional y la Pontificia Universidad Javeriana. Como resultado del proceso de revisión documental se encontraron 46 documentos, distribuidos así: 4 tesis de grado, 24 artículos, 8 libros, 3 estudios, 5 archivos, 1 revista y 1 artículo de prensa (figura 1).

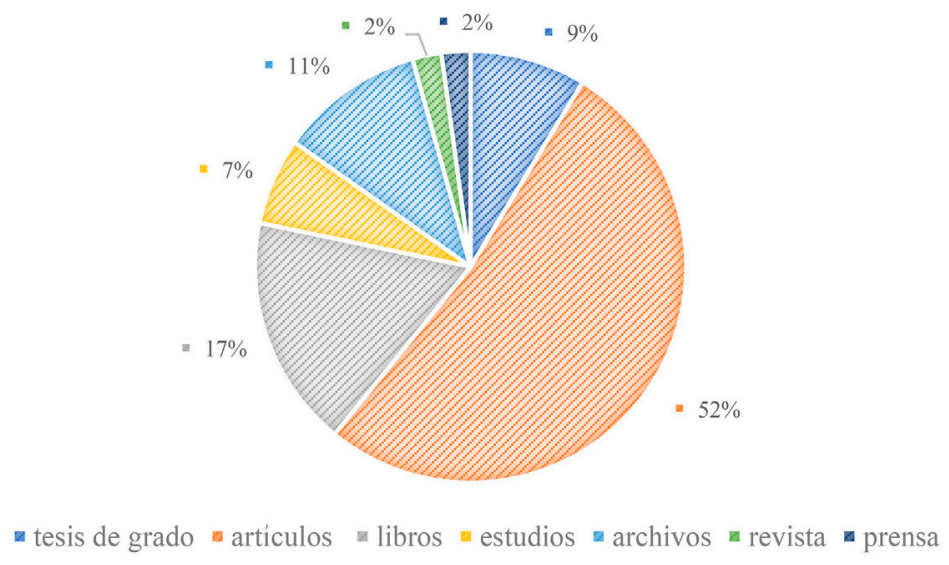

Figura 1. Balance cuantitativo de la tendencia investigativa de los documentos revisados Fuente: Elaboración propia

Se encontró que los dos temas de estudio más investigados son la inclusión y los roles desempeñados por la mujer militar. En Colombia, la mayoría de las investigaciones sociológicas militares se han centrado en comprender la interacción entre roles de género, el paternalismo generalizado, el sexismo y la influencia de las tradiciones culturales en la construcción de las identidades militares.

Algunos estudios han explorado la integración de las mujeres en el cuerpo logístico (Romo Obando, 2004; Garzón Ospina, 2014), la esperada participación de las mujeres de este cuerpo en los asuntos de defensa y seguridad (Pedraza Rozo 
\& Pardo Monroy, 2008; Díaz-Granados Manjarrés et al., 2017) y la participación de las mujeres en el conflicto armado colombiano (Rodríguez Reina et al., 2017). Sin embargo, estos estudios no abordan análisis desde los quehaceres de las oficiales según un enfoque de género.

\section{Orígenes de la integración de las mujeres en las Fuerzas Militares}

La inclusión de la mujer en distintos campos del poder político, militar, económico, religioso y sociocultural ha buscado reducir los posibles prejuicios de género. En el sector de seguridad y defensa nacional de Colombia, se han propiciado de manera progresiva las condiciones de equidad necesarias para que la mujer ingrese a la carrera de las armas. En efecto, de acuerdo con las cifras del Ministerio de Defensa Nacional (2016), para el 31 de diciembre del 2016, un total de 3.838 mujeres prestaron sus servicios como uniformadas en las FF. MM., distribuidas de la siguiente manera: 1.515 oficiales y suboficiales en el Ejército, 780 en la Armada Nacional y 1.038 en la Fuerza Aérea.

En el caso del Ejército Nacional, con la implementación del Decreto 2129 de 1976, se permitió el ingreso de las primeras doce mujeres a la carrera de las armas como, oficiales del cuerpo administrativo en calidad de profesionales en algunas áreas esenciales para la Fuerza como la medicina, la administración y el derecho.

El resultado de la incorporación de personal femenino en las FF. MM. ha sido positivo (Montańa, 2013), pues las mujeres han pasado de estar en cargos administrativos en la institución a ocupar labores del cuerpo logístico, inteligencia y comunicaciones, esto les ha permitido llegar al nivel de comandantes, con lo cual han demostrado que cuentan con las capacidades para desempeñarse en las funciones asignadas.

Con el tiempo, el Ejército Nacional ha integrado de forma progresiva personal femenino como oficiales de armas, con el fin de fortalecer las capacidades institucionales y proyectar una Fuerza incluyente, equitativa y altamente efectiva para el desarrollo de operaciones nacionales e internacionales.

$\mathrm{Al}$ respecto, oficiales de las armas son "todos aquellos formados, entrenados y capacitados con la finalidad principal de ejercer el mando y la conducción de los elementos de combate y apoyo del Ejército en todos los escalones de la jerarquía militar" (Decreto Ley 1790, 2000, art. 12). Por lo tanto, hay una clara diferencia con los oficiales administrativos en cuanto al campo de aplicación de sus funciones, así como de sus perfiles profesionales. 
Diez años después de la primera incorporación de mujeres oficiales de arma, preparadas como comandantes y líderes de pequeñas unidades de maniobra, no son pocos los espacios dentro de la institución que reconocen sus condiciones profesionales y su desempeño en cada uno de sus grados y cargos, gracias a lo cual se impulsa un cambio cultural para deslegitimar estereotipos y lograr posicionarse en un lugar importante dentro de la institución castrense. De acuerdo con Costantini (2008), con la inclusión de la mujer dentro de las FF. MM. a nivel mundial se contribuye al cierre de la brecha existente entre roles y funciones establecidos dentro de la doctrina militar vigente.

La doctrina Damasco, piedra angular del Ejército Nacional de Colombia, busca aumentar la interoperabilidad (estandarización, integración, cooperación y sinergia) de la Fuerza. Para esto, es fundamental un cambio cultural que facilite el reconocimiento de las capacidades del personal uniformado, sin distinción de género dentro de las diferentes armas, especialidades y unidades, lo cual es coherente con el desarrollo de las capacidades de las mujeres militares en beneficio de la institución militar.

Según Frieyro (2009), no se puede negar el apoyo de las mujeres en la guerra, tanto brindando ayuda médica como desempeñándose en apoyo de última instancia a las operaciones de combate. Bajo esta perspectiva, se crea el Comité de Mujeres en las Fuerzas de la OTAN, con el objetivo de conocer y mejorar la situación del personal femenino en los ejércitos de cada país (Agudo, 2011).

Fernández (2000) indica que fue en España, en 1979, que se propuso la creación de una división militar femenina como apoyo a las contingencias relacionadas principalmente con auxilio logístico. De ahí en adelante, la integración de las mujeres a las filas para ser asignadas a funciones de combate y acciones operativas afines, como la Logística, es un tema que tiene un alcance influenciado por la cultura militar de cada país, de acuerdo con las necesidades de los Ejércitos (Carreiras, 2017). Por su parte, Martín (2009) explica que la forma de incorporar mujeres al Ejército cambia según el criterio de cada país. Por ejemplo, en los años treinta, el servicio militar para las mujeres era común en el Reino Unido, Dinamarca y México, mientras que en otros países eran integradas como personal de tareas auxiliares. En los ańos cuarenta, las mujeres eran vinculadas como combatientes de primera línea en el ejército ruso y en Estados Unidos ingresaban con algunas limitaciones. 


\section{Tradiciones culturales y los roles de la mujer oficial del cuerpo logístico}

\section{Tradiciones}

Las tradiciones y estereotipos sobre el rol de género han estado asociadas a perspectivas tradicionales y a herencias culturales predominantemente masculinas. Diversos estudios han reportado las dificultades de las mujeres para integrar las FF. MM. (Matthews et al., 2009; Obradovic, 2016; Smith et al., 2018). La principal crítica realizada es que el proceso de integración de mujeres puede generar disparidad en las percepciones individuales y grupales, limitando las oportunidades y creando techos de cristal que obstaculizan el avance profesional.

En América Latina, quizás uno de los temas más desafiantes es el sexismo generalizado que prevalece en la sociedad y las organizaciones. Por ejemplo, los comportamientos tradicionales arraigados en la composición jerárquica de las familias fomentan una creencia sexista del paternalismo y la superioridad masculina que se aplica no solo dentro de los Ejércitos, sino también en las instituciones públicas y privadas (Panitz et al., 1983; Casas et al., 1994; Ruiz, 2005; García, 2009; Moradi \& DeBlaere, 2010; Bermúdez et al., 2015; Brown et al., 2018; Bermúdez Quintana et al., 2018; Garay Acevedo \& Guecha, 2018).

Algunos países con fuertes culturas sexistas han hecho esfuerzos continuos para superar tales estereotipos y han promovido el ingreso de mujeres en sus Ejércitos, para lo cual han creado estándares de evaluación neutrales, de forma que ellas puedan unirse a academias militares y tener igualdad de oportunidades para liderar unidades de combate y alcanzar rangos superiores. Estos esfuerzos han servido para mitigar los estereotipos y proporcionar a las mujeres una forma de demostrar sus capacidades y obtener el reconocimiento adecuado por sus logros, algo que la sociedad tradicional normalmente niega.

En Colombia, si bien las mujeres han servido como oficiales en el Ejército desde 1976, desde entonces fueron asignadas únicamente al cuerpo de Logística como médicas, abogadas y contadoras. Sin embargo, a partir del 2011 la Fuerza ha avanzado en la integración de las mujeres a funciones típicamente masculinas, como comandantes de pelotón, oficiales de Inteligencia o pilotos de helicóptero. Dentro de diferentes armas y especialidades se han vinculado de forma progresiva, iniciando con Comunicaciones e Inteligencia militar, en el 2011; Ingeniería, en el 2013; Aviación del Ejército, en el 2014; Artillería, en el 2016; e Infantería, en el 2018. 
Otros objetivos importantes que se han logrado en un corto período de tiempo están relacionados con la emisión de políticas de igualdad de género por parte del Ejército Nacional de Colombia, en 2017, y del Ministerio de Defensa, en 2018; políticas que promueven la participación de las mujeres en cursos de combate. Sin embargo, este ha sido un proceso lento.

\section{Roles}

Los roles de género son entendidos como las "actitudes y comportamientos esperados que una sociedad asocia con cada sexo" (Lindsey, 2015, p. 5), lo que los convierte en factores complejos dada su naturaleza, pues vinculan cogniciones y sentimientos relacionados con la internalización de la masculinidad y la feminidad (Robinson Kurpius \& Lucart, 2000, p. 215). Por tal motivo, configuran un determinante para avanzar hacia la igualdad de género y la inclusión en el Ejército colombiano.

Las principales dificultades del avance hacia una cultura de igualdad de género se enmarcan en las tradiciones arraigadas en culturas dominantes que limitan los roles y funciones de las mujeres. El enfoque tradicional promueve una continuación centrada exclusivamente en las labores asignadas a ellas por la sociedad: procreación, preparación de alimentos, cuidado de ancianos y niños, tareas domésticas, etc., todo lo cual limita sus posibilidades al hogar y no les permite incorporarse con éxito en ambientes masculinos. Esto último constituiría una perspectiva renovadora del rol de la mujer.

\section{Entre lo tradicional y lo renovador}

Para el caso militar se retoman las perspectivas tradicional y renovadora como ejes de análisis documental, y se incluyen las principales categorías conceptuales que describen los autores sobre género para este contexto (tabla 1).

Tabla 1. Perspectivas renovadora y tradicional en las categorías conceptuales asociadas a género en las FF. MM.

\begin{tabular}{lclcc}
\hline Autores & Año & \multicolumn{1}{c}{ Categoría } & $\begin{array}{c}\text { Perspectiva } \\
\text { tradicional }\end{array}$ & $\begin{array}{c}\text { Perspectiva } \\
\text { renovadora }\end{array}$ \\
\hline Luna & 2003 & Diferencias de género en educación & $\mathrm{x}$ \\
OTAN & 2016 & $\begin{array}{l}\text { Integración de las mujeres a las FF. } \\
\text { MM. para la efectividad e innova- } \\
\text { ción militar }\end{array}$ & $\mathrm{x}$ \\
\hline
\end{tabular}




\begin{tabular}{|c|c|c|c|c|}
\hline Autores & Año & Categoría & $\begin{array}{l}\text { Perspectiva } \\
\text { tradicional }\end{array}$ & $\begin{array}{l}\text { Perspectiva } \\
\text { renovadora }\end{array}$ \\
\hline Ellner & 2015 & Políticas de igualdad no exitosas & $\mathrm{x}$ & \\
\hline Trobaugh & 2018 & $\begin{array}{l}\text { Dificultad de vinculación por } \\
\text { entornos masculinos }\end{array}$ & & \\
\hline Smith y Rosenstein & 2017 & $\begin{array}{l}\text { Decisiones que impiden la promo- } \\
\text { ción en la carrera militar }\end{array}$ & $\mathrm{x}$ & \\
\hline Rodríguez Reina et al. & 2017 & $\begin{array}{l}\text { Participación de las mujeres en el } \\
\text { conflicto armado colombiano }\end{array}$ & $\mathrm{x}$ & $\mathrm{x}$ \\
\hline Montaña & 2013 & $\begin{array}{l}\text { Transición de las mujeres hacia } \\
\text { cargos de comandantes }\end{array}$ & & $\mathrm{x}$ \\
\hline Costantini & 2008 & $\begin{array}{l}\text { Inclusión de las mujeres en las } \\
\text { FF. MM. y doctrina militar }\end{array}$ & & $\mathrm{x}$ \\
\hline Frieyro & 2009 & $\begin{array}{l}\text { Apoyo de las mujeres en la guerra. } \\
\text { Última instancia }\end{array}$ & $\mathrm{x}$ & \\
\hline Martín & 2009 & $\begin{array}{l}\text { Mujeres como guerreros de } \\
\text { segunda categoría }\end{array}$ & $\mathrm{x}$ & \\
\hline Rosen et al. & 1996 & $\begin{array}{l}\text { Integración de mujeres en la } \\
\text { convivencia laboral. Tensión sexual }\end{array}$ & $\mathrm{x}$ & \\
\hline Bardera & 2009 & $\begin{array}{l}\text { Prejuicios y estereotipos que } \\
\text { limitan la participación de las } \\
\text { mujeres }\end{array}$ & $\mathrm{x}$ & \\
\hline Camacho Zambrano & 2011 & $\begin{array}{l}\text { Las FF. MM. como un espacio } \\
\text { militar no apto }\end{array}$ & $\mathrm{x}$ & \\
\hline
\end{tabular}

Fuente: Elaboración propia

Las relaciones de poder, jerarquía y dominación obstaculizan la integración apropiada de la mujer en las FF. MM. (Goldstein, 2001). No obstante, en momentos de necesidad de recursos humanos, la presencia femenina ha sido bienvenida en puestos de apoyo o escenarios de combate (DeLance, 2016). Durante siglos, la actividad militar se ha considerado como propia de varones, lo que excluye a las mujeres colocándolas como guerreros de segunda categoría o en 
el más común de los casos, como seres a los que hay que sobreproteger y dejarles las tareas más sencillas, porque se cree que son incapaces de dirigir una operación militar (Martín, 2009).

Bardera (2009) complementa este concepto al sugerir que existe una serie de prejuicios tanto biológicos como fisiológicos que estereotipan a la mujer y hacen que esta sea relegada y se le impida demostrar su capacidad activa en operaciones de logística y combate. Por lo cual, según Oliva (2005) se da lugar a una situación de patriarcado dentro de las líneas militares, en forma de una autoridad que establece la división del trabajo dentro de la actividad militar según el género de la persona.

Por otro lado, Rosen et al. (1996) afirman que existen especialistas en el tema que consideran que la integración de las mujeres en las FF. MM. disminuye la efectividad en las operaciones y otras labores propias del ejército, por la tensión sexual que ocasiona en la convivencia laboral.

Mientras la incorporación militar de las mujeres es un fenómeno habitual en los ejércitos de países como Chile, Brasil, Francia, Estados Unidos o España ("Primeras mujeres militares colombianas, listas para participar en la guerra”, 2009), para el caso colombiano este hecho se reviste de una mayor relevancia por tratarse de un territorio castigado desde hace más de cuatro décadas por un conflicto armado. En relación con esto, Cuenca (2011) señala que, en el contexto del conflicto colombiano, las mujeres militares ganaron espacio desarrollando tareas muy importantes, como facilitar el acercamiento a la comunidad en las zonas rurales donde operan algunas unidades militares, manejando los medios de comunicación, dirigiendo los programas de difusión a través de esos medios e incluso escribiendo en las publicaciones de las Fuerzas sobre temas relacionados con los derechos humanos.

Si bien el ingreso de las mujeres al Ejército podría considerarse una decisión con enfoque renovador, los quehaceres de las oficiales del cuerpo logístico se encasillan en aspectos instrumentales de tipo tradicional, como proveer asistencia a las unidades de combate y ocuparse de aspectos administrativos que no son desempeñados por personal masculino apto para el combate. Así las cosas, se han generado tensiones al interior de la institución respecto a las relaciones entre géneros, principalmente, entre los hombres, porque generalmente pensaban que ese no era un espacio para las mujeres (Ejército Nacional, 1998b; Camacho Zambrano, 2011; Fernández-Osorio \& Latorre Rojas, 2018).

Este contexto se han configurado barreras sociales que no permiten la integración completa de las mujeres con las funciones renovadoras dentro de la institución (Ejército Nacional, 1998a), por lo cual se han reportado acciones de abuso de poder sobre el personal femenino (Ejército Nacional, 1998b). Dicha situación 
se presenta, en parte, debido a que las mujeres fueron integradas al Ejército sin una planeación adecuada, tanto de su vida laboral como de la forma de relacionarse con el personal masculino en la institución, lo que ha generado una brecha social de género (Ejército Nacional, 2015).

\section{Los quehaceres de las mujeres oficiales del cuerpo logístico}

Cruz (2005) se refiere a la polémica que ha suscitado el ingreso y la participación de las mujeres en las fuerzas armadas, ya que se considera que es cosa de hombres y que la participación de las mujeres solo debe existir para asuntos tradicionales de corte administrativo, no para las funciones de combate. Las razones esgrimidas hacen referencia a que el combate armado requiere esencialmente la fuerza física y la destreza de los hombres. También se insiste en que en el ambiente militar las mujeres son consideradas como una población que demuestra fragilidad, sensibilidad para hacer cumplir las políticas o simplemente ausencia de carácter fuerte para llevar a cabo acciones que demuestren el uso adecuado de la fuerza (Ejército Nacional, 1998b).

Por lo anterior, los quehaceres de la mujer del cuerpo logístico del Ejército Nacional se han equiparado con los de las oficiales del cuerpo administrativo de la misma arma. Si bien los dos tipos de oficial se desempeñan en el mismo rol, existen diferencias sustanciales que radican en su formación. Las oficiales del cuerpo logístico de arma se ubican más en un enfoque renovador, pues ejercen el liderazgo militar y comandan unidades conformadas generalmente por hombres, para garantizar el apoyo al movimiento y la maniobra en áreas adelantadas de combate y, así, cumplir la misión institucional. Por otra parte, las oficiales del cuerpo administrativo han sido capacitadas con un enfoque tradicionalista: desempeñar su profesión en áreas atrasadas o en la retaguardia del campo de batalla, sin mando de unidades y sin responsabilidades operacionales directas. Esto conlleva a que, aunque todas las mujeres oficiales del cuerpo logístico tengan la capacidad para dirigir operaciones militares, sus capacidades se vean opacadas por aspectos culturales dentro de la Fuerza, lo que las relega a cargos administrativos, de forma que se afectan su desempeño en la institución y su proyección en la carrera.

Camacho Zambrano (2014) revela la percepción de los hombres frente a las mujeres en la Escuela Militar de Cadetes "General José María Córdova" en un ambiente predominantemente masculino, que discrimina el quehacer de las 
mujeres militares, principalmente por sus características morfofisiológicas, como la complexión muscular y su nivel de fuerza para manejar peso. Esto puede significar un inconveniente para una unidad militar, por esa razón se les ubica en labores administrativas o de logística. Esta situación ocurre porque el centro de formación militar fue creado con base en las capacidades de los hombres, lo que es tradicionalista, pero no fue pensado para el ingreso de mujeres.

De forma complementaria, la investigación de Husain-Talero y Muñoz (2018) indica que el personal militar masculino se resiste a los cambios generados por la inclusión de la mujer en la institución, ya que existe la concepción de que la mujer no tiene la misma condición física que el hombre para el entrenamiento en combate. De hecho, se destacan en este estudio las agresiones verbales de las que son víctimas las mujeres respecto a sus capacidades físicas e intelectuales, y el trato diferenciado por parte de sus superiores (Husain-Talero \& Muñoz, 2018).

Adicionalmente, Williams (2009) afirma que las mujeres del cuerpo logístico tienen un rol especial no solo como soldado, sino como asistente social en las regiones de posconflicto, ya que ellas tienen facilidad para empatizar con la gente y llevar a cabo iniciativas de cooperación social y de promoción del talento humano.

En una apuesta por la renovación, las FF. MM. y el Ejército Nacional han modificado sus normas de carrera para que las mujeres puedan alcanzar cada uno de los grados del escalafón militar, incluido el grado de general. Esta modificación pretende una mayor inmersión del personal femenino en asuntos concernientes al desarrollo de actividades militares, como se refiere en el documento Política de defensa y seguridad: todos por un nuevo país del Ministerio de Defensa (2016, p. 4), en el que se resalta el papel de la mujer en igualdad de condiciones al hombre en todos los ámbitos de las FF. MM.

Así mismo, para mitigar el impacto negativo de las tradiciones culturales en los roles y funciones de las mujeres militares $-\mathrm{y}$, por ende, en el alistamiento de la Fuerza—, se creó en el año 2012 el primer Observatorio de Género del Ejército Nacional, que trabaja en el diseño e implementación de políticas dirigidas a incluir y preservar la equidad de género en la institución. La más reciente política del Observatorio, denominada Atenea, busca acelerar el cambio cultural en la institución armada, modelando una cultura de equidad de género para eliminar los prejuicios hacia las mujeres, como el de que estas no tiene las mismas habilidades que los hombres y, por ello, deben recibir un trato diferencial. 


\section{Conclusiones}

Este estudio sobre las mujeres oficiales del cuerpo logístico del Ejército Nacional ofrece resultados que permiten mejorar el desempeño de sus roles y funciones, de acuerdo con sus capacidades y no con su género. No obstante, es claro que la dinámica entre hombres y mujeres en las FF. MM. aún requiere de mayores estudios para determinar las estrategias necesarias para fomentar la equidad de género.

A pesar de existir políticas de equidad y de inclusión femenina, persiste la discriminación hacia la mujer en cuanto a su desempeño físico. Aunque todas las mujeres están en la capacidad de dirigir operaciones militares, sus posibilidades se ven opacadas por creencias tradicionalistas socialmente heredadas. Actualmente, dentro de la institución —en especial, en el cuerpo logístico- persiste este último enfoque, pues se procura que la mujer asuma tareas administrativas, pero no operativas, que corresponden al personal masculino por fuerza y destreza. En el Ejército Nacional es un tema de revisión la inclusión progresiva de la mujer en operaciones de combate de primera línea.

Esta revisión se destaca como una gran oportunidad para que la Fuerza evalúe de forma diferencial los roles de las oficiales del cuerpo logístico y las desligue de sus funciones exclusivamente administrativas. Así mismo, se resalta la necesidad de un Ejército más flexible, dispuesto a impulsar la igualdad de género para fortalecer la democracia y los derechos humanos.

Debido a que la incorporación de mujeres a las filas de los distintos Ejércitos del mundo ha sido objeto de múltiples estudios, este artículo abre las puertas a nuevas líneas de investigación en las que se explore la participación activa de las mujeres oficiales del cuerpo logístico en cargos que no sean administrativos dentro de la Fuerza.

\section{Referencias}

Agudo, Y. (2011). Historia del reconocimiento público de las mujeres. Géneros 18(9), 167-194.

Bardera, M. P. (2009). Perfil psicosocial de la mujer militar. En M. Gómez, \& I. Sepúlveda (Eds.), Las mujeres militares en España 1988-2008 (pp. 222-251). Instituto Universitario General Gutiérrez Mellado.

Bermúdez Quintana, E., Valero Londońo, A., Díaz Chavarro, B., Castrillón Muñoz, E., \& Castrillón Libreros, D. (2018). Perpetuación de violencias verbal y psicológicas en las relaciones afectivas y de familia en adolescentes de Cali. Revista Científica General José María Córdova, 16(21), 61-80. https://doi.org/10.21830/19006586.312 
Bermúdez, M., Sharp, E., \& Taniguchi, N. (2015). Tapping into the complexity: Ambivalent sexism, dating, and familial beliefs among young Hispanics. Journal of Family Issues, 36(10), 1274 1295. https://doi.org/10.1177/0192513X13506706

Brown, R., Baughman, K., \& Carvallo, M. (2018). Culture, masculine honor, and violence toward women. Personality and Social Psychology Bulletin, 44(4), 538-549. https://doi. org/10.1177/0146167217744195

Camacho Zambrano, C. (2011). Políticas de equidad de género en las Fuerzas Armadas. Impactos en la cultura institucional militar: el caso de la incorporación de cadetes femeninas en la ESMIC. Revista Cientifica General José Maria Córdova, 9(9), 69-94. https://doi. org/10.21830/19006586.246

Camacho Zambrano, C. (2014). Ejército, feminidades y géneros performativos: las experiencias de ser mujer y militar en la Escuela Militar de Cadetes "General José María Córdova" [Tesis de maestría, Pontificia Universidad Javeriana]. Repositorio Institucional. https://repository.javeriana. edu.co/handle/10554/14323

Carreiras, H. (2017). Militares y perspectiva de género: la mujer en las Fuerzas Armadas de las democracias occidentales. Universidad de la Defensa Nacional.

Casas, M., Wagenheim, B., Banchero, R., y Mendoza-Romero, J. (1994). Hispanic masculinity: Myth or psychological schema meriting clinical consideration. Hispanic Journal of Behavioral Sciences, 16(3), 315-331. https://doi.org/10.1177/07399863940163009

Cheatham, H. (1984). Integration of women into the U.S. Military. Sex Roles, 1(2), 141-153. https://doi.org/10.1007/BF00287445

Collins-Dogrul J., \& Ulrich, J. (2018). Fighting stereotypes. Armed Forces \& Society, 44(3), 436-559. https://doi.org/10.1177/0095327X17715650

Costantini, P. (2008). La más asombrosa arma moderna del arsenal occidental. La Aljaba.

Cruz, J. (2005). Reflexiones sobre la presencia de la mujer en los ejércitos desde una perspectiva histórico-jurídica. En Mujer, fuerzas armadas y conflictos bélicos: una visión panorámica (pp. 143-184). Ministerio de Defensa; Instituto Español de Estudios Estratégicos.

Cuenca, Y. (2011). La participación de algunas mujeres en el Ejército Nacional de Colombia. La Manzana de la Discordia, 6 (2), 57-63.

DeGroot, G. (2001). A few good women: Gender stereotypes, the military and peacekeeping. International Peacekeeping, 8(2), 23-38. https://doi.org/10.1080/13533310108413893

DeLance, L. (2016). Women in combat. En N. Naples (Ed.), The Wiley Blackwell Encyclopedia of Gender and Sexuality Studies. Wiley. https://doi.org/10.1002/9781118663219.wbegss289

Díaz-Granados Manjarrés, J., Santo Quiñonez, C., \& Ortiz Gómez, K. (2017). Transformación del Ejército al incorporar mujeres de arma. Escuela Militar de Cadetes "General José María Córdova".

Egnell R., \& Alam, M. (2019). Women and gender in the military: An international comparison. Georgetown University Press.

Egnell, R., Hojem, P., \& Berts, H. (2014). Gender, military effectiveness, and organizational change: The Swedish model. Palgrave MacMillan.

Ejército Nacional. (1998a). Archivos de la oficina del servicio psicológico. Imprenta del Ejército.

Ejército Nacional. (1998b). Archivos de la oficina de trabajo social. Imprenta del Ejército. 
Ejército Nacional. (2015). Mujer militar: igualdad y liderazgo. https://www.ejercito.mil.co/?idcategoria $=376416$

Ellner, A. (2015). The ethics of inclusion: Gender integration, equal opportunity, and sexual assault in the Australian, British, Canadian and U.S. Armed Forces. En G. Lucas (Ed.), The Routledge handbook of military ethics (pp. 300-317). Routledge.

Fernández, F. (2000). La mujer y las fuerzas armadas: un estudio jurídico-constitucional. En Mujer y constitución en España (pp. 295-334). Centro de Estudios Políticos y Constitucionales.

Fernández-Osorio, A., \& Latorre Rojas, E. (2018). La construcción del rol de la mujer militar. Escuela Militar de Cadetes "General José María Córdova”.

Frieyro, B. (2009). La mujer soldado en España. En M. Gómez, \& I. Sepúlveda (Ed.), Las mujeres militares en España 1988-2008 (pp. 173-199). Instituto Universitario General Gutiérrez Mellado.

Garay Acevedo, C., \& Guecha, A. (2018). Los derechos humanos en Colombia. Revista Cientifica General José María Córdova, 16(23), 83-105. https://doi.org/10.21830/19006586.307

García, L. (2009). "Now why do you want to know about that?": Heteronormativity, sexism, and racism in the sexual (mis)education of Latina youth. Gender and Society, 23(4), 520-541. https://doi.org/10.1177/0891243209339498

Garzón Ospina, L. (2014). Una mirada al género y conexiones con el ámbito militar: estado del arte 20042014. Escuela Militar de Cadetes "General José María Córdova”.

Goldstein, J. S. (2001). War and gender. How gender shapes the war system and vice versa. Cambridge University Press.

Husain-Talero, S., \& Muñoz, L. (2018). Caracterización del liderazgo femenino en ámbitos militares. Reflexiones a partir de las experiencias de las alféreces en su fase de mando. Cadernos Pagu, 56, 1-29. http://dx.doi.org/10.1590/18094449201900560009

Karazi-Presler, T., Sasson-Levy, O., \& Lomsky-Feder, E. (2018). Gender, emotions management, and power in organizations: The case of Israeli women junior military officers. Sex Roles, 78(8), 573-586. https://doi.org/10.1007/s11199-017-0810-7

King, A. (2016). The female combat soldier. European Journal of International Relations, 22(1), 122-43. https://doi.org/10.1177/1354066115581909

Larwood, L., Glasser, E., \& McDonald, R. (1980). Attitudes of male and female cadets toward military sex integration. Sex Roles, 6(3), 381-390. https://doi.org/10.1007/BF00287359

Lindsey, L. (2015). Gender roles: A sociological perspective. Routledge.

Luna, L. (2003). La historia feminista del género y la cuestión del sujeto. Boletín Americanista, 52, 105-121.

Martín, V. (2009). Las mujeres en los ejércitos europeos y americanos. En M. Gómez, \& I. Sepúlveda (Eds.), Las mujeres militares en España 1988-2008 (pp. 23-43). Instituto Universitario General Gutiérrez Mellado.

Matthews, M., Ender, M., Laurence, J., \& Rohall, D. (2009). Role of group affiliation and gender on attitudes toward women in the Military. Military Psychology, 21(2), 241-251. https://doi. org/10.1080/08995600902768750

Ministerio de Defensa Nacional. (2016). Las mujeres en las Fuerzas Militares.

Montańa, P. (2013). Evolución histórica del papel de la mujer en el ejército colombiano. Revista Armas y Letras, 57, 68-71. 
Moradi, B., \& DeBlaere, C. (2010). Women's experiences of sexist discrimination: Review of research and directions for centralizing race, ethnicity, and culture. En H. Landrine, \& N. Russo (Eds.), Handbook of diversity in feminist psychology (pp. 173-210). Springer Publishing Co.

Obradovic, L. (2016). Gender integration in NATO military forces. Cross-national analysis. Ashgate Publishing, Ltd.

Oliva, A. (2005). Debates sobre el género. En C. Amorós, \& A. de Miguel (Eds.), Teoría Feminista de la ilustración a la globalización (pp. 15-60). Minerva.

OTAN. (2016). Summary of the National Reports of NATO Member and Partner Nations to the NATO Committee on Gender Perspectives. OTAN.

Panitz, D., McConchie, S., Sauber, R., \& Fonseca, J. (1983). The role of machismo and the Hispanic family in the etiology and treatment of alcoholism in Hispanic American males. The American Journal of Family Therapy, 11(1), 31-44. https://doi.org/10.1080/01926188308250109

Pedraza Rozo, E., \& Pardo Monroy, J. (2008). Análisis Histórico y proyección de la participación de la mujer en la Defensa Nacional. Escuela Militar de Cadetes "General José María Córdova".

Presidencia de la República de Colombia. (14 de septiembre de 2000). Por el cual se modifica el Decreto que regula las normas de carrera del personal de oficiales y suboficiales de las Fuerzas Militares. [Decreto Ley 1790 de 2000].

Primeras mujeres militares colombianas, listas para participar en la guerra. (2009). Vanguardia. http://goo.gl/gzzpVb

Robinson Kurpius, S., \& Lucart, A. (2000). Military and civilian undergraduates: Attitudes toward women, masculinity, and authoritarianism. Sex Roles, 43(3-4), 255-265. https://doi. org/10.1023/A:1007085015637

Rodríguez Reina, A., Virviescas Martínez, S., \& Pulgarín Sánchez, M. (2017). El rol de la mujer en el conflicto armado interno colombiano: desde experiencias de combatientes. Escuela Militar de Cadetes "General José María Córdova".

Rohall, D., Ender, M., \& Matthews, M. (2006). The effects of military affiliation, gender, and political ideology on attitudes toward the wars in Afghanistan and Iraq. Armed Forces \& Society, 33(1), 59-77. https://doi.org/10.1177/0095327X06289817

Romo Obando, M. (2004). La mujer y su importancia en el Ejército de Colombia. Escuela Militar de Cadetes "General José María Córdova”.

Rosen, L. N., Durand, D. B., Bliese, P. D., Halverson, R. R., Rothberg, J. M., \& Harrison, N. L. (1996). Cohesion and readiness in gender-integrated combat service support units: The impact of acceptance of women and gender ratio. Armed Forces \& Society, 22(4), 537-553. https://doi.org/10.1177/0095327X9602200403

Ruiz, E. (2005). Hispanic culture and relational cultural theory. Journal of Creativity in Mental Health, 1(1), 33-55. https://doi.org/10.1300/J456v01n01_05

Smith, D., \& Rosenstein, J. (2017). Gender and the Military Profession. Armed Forces \& Society, 43(2), 260-279. https://doi.org/10.1177/0095327X15626722

Smith, D., Rosenstein, J., Nikolov, C., \& Chaney, A. (2018). The power of language: Gender, status, and agency in performance evaluations. Sex Roles, 80(2), 1-13. https://doi.org/10.1007/ s11199-018-0923-7 
Trobaugh, E. (2018). Women, regardless: Understanding gender bias in U.S. military integration. Joint Force Quarterly, 88, 1-12.

Verveer, M. (2019). Foreword. En R. Egnell, \& A. Mayeshe (Eds.), Women and gender perspectives in the military (pp. v-viii). Georgetown University Press.

Williams, J. (2009) How hidden bias affects everyday workplace interactions. En J. Williams (Ed.), Fair Measure: Toward Effective Attorney Evaluations (pp. 15-79). American Bar Association Commission on Women in the Profession. 